\title{
Algal Flora and Ecology of the High Mountain Lakes in the Artabel Lakes Nature Park (Gümüşhane, Turkey), I- Bacillariophyta
}

\author{
Bülent Şahin ${ }^{1}$, Bülent Akar ${ }^{2}$, Sophia Barınova ${ }^{3 *}$ \\ ${ }^{1}$ Department of Biology Education,Fatih Education Faculty,Trabzon University, 61335 Söğ̈̈tlü-Trabzon, \\ Turkey \\ ${ }^{2}$ Department of Food Engineering, Faculty of Engineering and Natural Sciences, Gümüşhane \\ University, Gümüşhane, Turkey \\ ${ }^{* 3}$ Institute of Evolution, University of Haifa, Mount Carmel, 199 Abba Khoushi Ave., Haifa 3498838, Israel \\ *Corresponding Author: Sophia Barınova, Institute of Evolution, University of Haifa, Mount Carmel, \\ 199 Abba Khoushi Ave., Haifa 3498838, Israel.
}

\begin{abstract}
The species composition of benthic diatoms of 18 selected high mountain lakes in the Artabel Lakes Nature Park of Turkey were investigated (on 15 August 2013 and on 13 August 2016). In all, 95 species of diatoms belonging to 46 genera were recorded. The most species rich genera are Pinnularia (15), Eunotia (6) and Gomphonema (6), while other genera are presented with one or more species. Only Eunotia praerupta and Pinnularia interrupt are considered as very frequent (VF) (2.10\%) species. The diatom flora of the Artabel Lakes Nature Park was formed by cosmopolitan species with a large influence from species in alpine and subalpine area. A comparison of the benthic assemblages of the investigated lakes showed differences in both in relative abundance and species present in the individual lakes. The physico-chemical conditions of the studied waters also provided a suitable environment for the development of the diatom flora. The Willis curve of the diatom flora in the Artabel Lakes Nature Park was determined to be close to a hyperbolic shape. This study provides the groundwork for future researches.
\end{abstract}

Keywords: benthic diatoms, high mountain lakes, Artabel Lakes Nature Park, Turkey

\section{INTRODUCTION}

Benthic algae are one of the living groups that constitute the primary producers in aquatic ecosystems and they are also the main energy source in the food chain for aquatic organisms [1]. Diatoms are one of the important groups in water ecosystems and form a large part of the benthic communities (often 90-95\%) [2].They are also very important indicators of environmental changes and are used in the biological monitoring of water ecosystems [3]. Therefore, it is of great significance to describe their prevalence and ecology [4].

In many parts of the world, there are mountain lakes in high altitudes in nature conservation areas that are still and are relatively far from anthropogenic impacts. They can reveal important information on biodiversity conservation, recreation, and storage of good-quality water [5]. In Turkey, Artabel Lakes Nature Park is an important protected area and has 23 high mountain lakes. Unfortunately, there is only one published data about the algal flora of these lakes [6].Therefore, the investigation of algal flora of the park is very important both for Turkey and the world.

The basic aim of this study was to identify the floristic and ecological of the diatom assemblages inhabiting the lakes in the Artabel Lakes Nature Park.

\section{Materials AND Method}

\subsection{Description of Study Site}

The Artabel Lakes Nature Park is an important protected area of Turkey. It was declared as a nature park in 1998. It is within the borders of Torul County of the Gümüşhane province of the Eastern Black sea region. It is located between 40 $21^{\prime} 36^{\prime \prime}-40^{\circ} 26^{\prime} 42^{\prime \prime}$ north and $39^{\circ} 0^{\prime} 24^{\prime \prime}-39^{\circ} 8^{\prime} 23^{\prime \prime}$ east. The area of Artabel Lakes Nature Park is 5859 hectares (Fig.1) The Artabel Lakes Nature Park includes terrestrial and aquatic ecosystems with rich biodiversity and has endemic species listed in the International Nature Conservation Union (IUCN) and the Berne Convention Annex I, II and III [7]. 


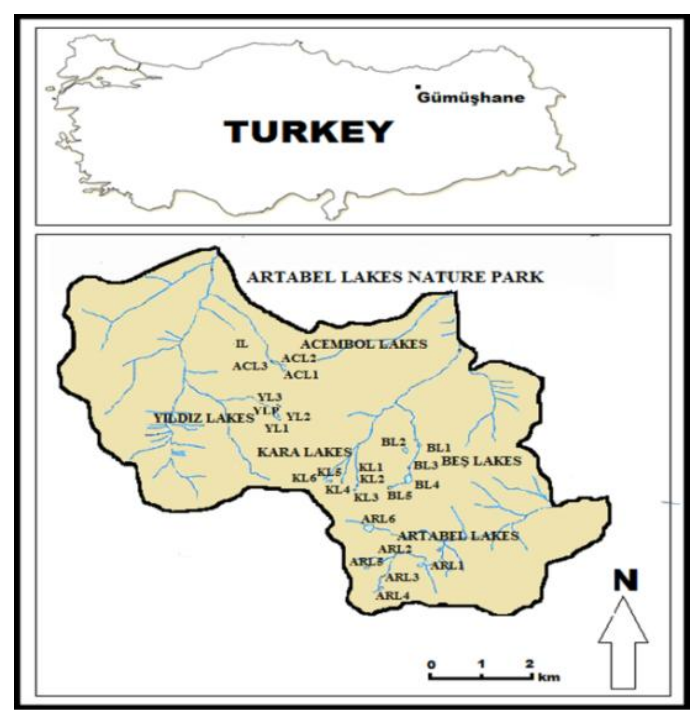

Figure 1. The Studied Area [7]

In the Artabel Lakes Nature Park, there are three stream basins, which are the Gümüştuğ Stream basin (Kara and Beş Lakes), the Artabel Stream basin (Artabel Lakes), and the Kongel Stream basin (Yıldız and Acembol Lakes). The total basin area is approximately $58.2 \mathrm{~km}^{2}$ and is composed of 5 different lake sites including the Artabel Lakes (ARL), Acembol Lakes (ACL), Beş Lakes (BL), Kara Lakes (KL) and Yıldız Lakes (YL). In total, there are 23 lakes: the Artabel Lakes (6), Acembol Lakes (3), Beş Lakes (5), Kara Lakes (6) and Yıldız Lakes (3). Some of the different size of the lakes have been linked to othersor are independence of each other [7]. There is also a previously unnamed lake (Isimsiz Lake: IL) and a small pond (Y1ld1z Lakes Pond (YLP)).These lakes are glacial in origin, covered with iced layers for at least 8 months of the year and situated in the alpine zone (2687-3030 m a.s.l.).It is not possible to fully describe the diatom flora year-round because of difficulties surrounding the access to these areas.

The aim of this study was to investigate 17 lakes and one pond situated at different sea levels (2687$2980 \mathrm{~m}$ a.s.l.) and characterizes its flora and environment on the base of physical and chemical parameters of the waters as well as epipelic, epilithic, and epiphytic diatom algae.

\subsection{Sampling and Laboratory Studies}

The Artabel Lakes Nature Park was visited on two different dates (on 15 August 2013 and on 13 August 2016). The diatom samples were collected from three different habitats: sediments (epipelic), stones (epilithic), mosses, and macro algae (epiphytic). The Kara Lakes could not be visited because the terrain was too difficult to traverse. Also, there was no water in the BL5 Lake; therefore, diatom and water samples could not be taken. Epipelic samples were collected with a glass tube from the surface of the sediment at all water bodies, except Lake BL2. Epilithic samples were taken once from the littoral zone of ARL1, ARL2, BL2, ACL1, ACL2, ACL3 and IL lakes. The epilithic diatoms were scraped from randomly chosen stones with the toothbrush. The samples were then washed and placed into plastic bottles. Epiphytic diatoms were collected by squeezing out mosses (Hygrohypnum luridum (Hedw.) Jenn.), and filamentous algae (Microspora sp.) From the ARL1, ARL3, YL1, YL2, YL3, ACL2, ACL3, IL lakes, and YLP Pond. All algological materials were preserved in 4\% formaldehyde. Dissolved oxygen, water temperature, $\mathrm{pH}$ and conductivity were measured with portable devices Orion-4Star and YSI-55 at each sampling site.

A total of 43 samples were analyzed. Hydrogen peroxide technique to clean diatoms frustules off [8] and mounted in Naphrax®. Diatoms were identified using a Leica DM 2500 microscope and were photographed with a Leica DFC 290 camera attached to the microscope. Diatoms were identified with help of international handbooks [9-20]. The validity of the species name was checked from Algae Base [21].

Frequencies of algal taxa were determined according to the following scale based on the number of lakes studied in the Artabel Lakes Nature Park. Very rare (VR): taxa recorded in 1-20\% of investigated lakes; rare (R): taxa recorded in 21-40\% of investigated lakes; common (C): taxa 
Algal Flora and Ecology of the High Mountain Lakes in the Artabel Lakes Nature Park (Gümüşhane, Turkey), I- Bacillariophyta

recorded in 41-60\% of investigated lakes; frequent $(\mathrm{F})$ : taxa recorded in $61-80 \%$ of investigated lakes; very frequent $(\mathrm{VF})$ : taxa recorded in $81-100 \%$ of investigated lakes [22].

\section{RESULTS AND DISCUSSION}

\subsection{Physical and Chemical Analysis}

The results of physico-chemical analysis of the studied waters are given in other paper [23]. From these results, it can be concluded that the waters are characterized by fewer temperature fluctuations (10.1-19.1 ${ }^{\circ} \mathrm{C}$ ). According to values of $\mathrm{pH}$ of the examined waters, it can be said that the waters of the Artabel Lakes Nature Park are acidic and circumneutral $(\mathrm{pH}$ 6.19-7.52). The concentrations of dissolved oxygen (DO) and total dissolved matter (TDS) varied from 2.10 to $9.45 \mathrm{mg} / \mathrm{L}$ and 6 to $30.55 \mathrm{mg} / \mathrm{L}$, respectively. The values of water conductivity (C) of the studied waters can be characterized as low (12.0-49.9 $\mu \mathrm{S} / \mathrm{cm})$. Measured environmental variables as well as diatom species richness are represented in Fig. 2 where all parameters are organized according to altitude of habitats. Therefore, it was observed that the water $\mathrm{pH}$ was rather stabile, but temperatures slightly decreased with altitude. It was intersting to find that water variables dissolved oxygen and conductivity had a tendency to increase in the middle altitude interval, whereas total dissolved solids and species richness decreased.

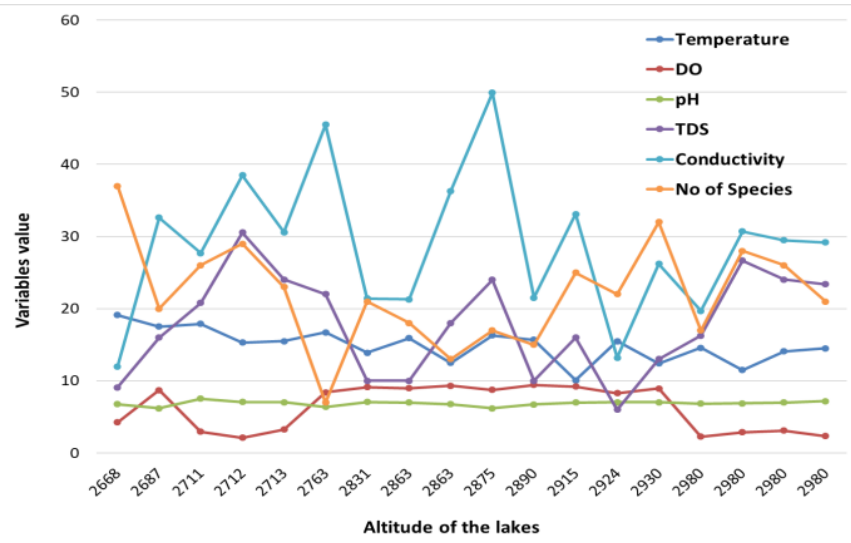

Figure 2. Distribution of water variables and number of species in the examined lakes of the Artabel Lakes Nature Park according to altitude.

In Table 1 a linear, negative, high level relationship between altitude and water temperature $(\mathrm{T})(\mathrm{r}=-$ $0.701 ; \mathrm{p}<0.01)$ can be seen. This means that as the altitude of the lakes increases, the temperature decreases. There is also a linear, negative, moderate relationship between total dissolved solids (TDS) and dissolved oxygen (DO) $(\mathrm{r}=-0.574 ; \mathrm{P}<0.01)$. This finding suggests that as the amount of total dissolved solids increases, the amount of dissolved oxygen decreases. In addition, it is seen that there is a linear, positive, high-level relationship between total solids (TDS) and conductivity (C) ( $\mathrm{r}=$ $0.738 ; \mathrm{P}<0.01)$. This means that as the amount of total dissolved solids increases, the conductivity increases.

Table 1. Correlations among several morphological, physical, chemical and biological variables of the studied lakes

\begin{tabular}{|c|c|c|c|c|c|c|c|c|}
\hline & & $\begin{array}{l}\text { Altitude } \\
\text { (m a.s.l) }\end{array}$ & $\begin{array}{c}\mathrm{T} \\
\left({ }^{\circ} \mathrm{C}\right)\end{array}$ & $\begin{array}{c}\mathrm{DO} \\
(\mathrm{mg} / \mathrm{L})\end{array}$ & $\mathrm{pH}$ & $\begin{array}{c}\text { TDS } \\
(\mathrm{mg} / \mathrm{L})\end{array}$ & $\begin{array}{c}\mathrm{EC} \\
(\mu \mathrm{S} / \mathrm{cm})\end{array}$ & $\begin{array}{c}\text { Number } \\
\text { of species }\end{array}$ \\
\hline \multirow{3}{*}{$\begin{array}{l}\text { Altitude } \\
\text { (m } \\
\text { a.s.l) }\end{array}$} & $\mathrm{N}$ & & 18 & 18 & 18 & 18 & 18 & 18 \\
\hline & $\mathrm{r}$ & & -0.701 & 0.018 & 0.065 & -0.037 & -0.103 & -0.172 \\
\hline & $p$ & & $0.002 *$ & 0.943 & 0.797 & 0.884 & 0.684 & 0.495 \\
\hline \multirow{3}{*}{$\begin{array}{c}\mathrm{T} \\
\left({ }^{\circ} \mathrm{C}\right)\end{array}$} & $\mathrm{N}$ & 18 & \multirow{3}{*}{-} & 18 & 18 & 18 & 18 & 18 \\
\hline & $\mathrm{r}$ & -0.701 & & -0.163 & -0.254 & -0.113 & -0.122 & 0.040 \\
\hline & $p$ & $0.002 *$ & & 0.518 & 0.310 & 0.656 & 0.630 & 0.876 \\
\hline \multirow{3}{*}{$\begin{array}{c}\mathrm{DO} \\
(\mathrm{mg} / \mathrm{L})\end{array}$} & $\mathrm{N}$ & 18 & 18 & \multirow[t]{3}{*}{ 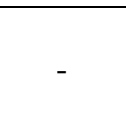 } & 18 & 18 & 18 & 18 \\
\hline & $r$ & 0.018 & -0.163 & & -0.223 & -0.574 & 0.088 & -0.371 \\
\hline & $p$ & 0.943 & 0.518 & & 0.374 & $0.009 *$ & 0.728 & 0.130 \\
\hline \multirow{2}{*}{$\mathrm{pH}$} & $\mathrm{N}$ & 18 & 18 & 18 & \multirow[t]{2}{*}{ 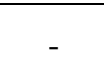 } & 18 & 18 & 18 \\
\hline & $\mathrm{r}$ & 0.065 & -0.254 & -0.223 & & 0.124 & -0.110 & 0.435 \\
\hline
\end{tabular}


Algal Flora and Ecology of the High Mountain Lakes in the Artabel Lakes Nature Park (Gümüşhane, Turkey), I- Bacillariophyta

\begin{tabular}{|c|c|c|c|c|c|c|c|c|}
\hline & $\mathrm{p}$ & 0.797 & 0.310 & 0.374 & & 0.624 & 0.664 & 0.071 \\
\hline \multirow{3}{*}{$\begin{array}{c}\text { TDS } \\
(\mathrm{mg} / \mathrm{L})\end{array}$} & $\mathrm{N}$ & 18 & 18 & 18 & 18 & \multirow{3}{*}{ - } & 18 & 18 \\
\hline & $\mathrm{r}$ & -0.037 & -0.113 & -0.574 & 0.124 & & 0.738 & -0.038 \\
\hline & $\mathrm{p}$ & 0.884 & 0.656 & $0.009 *$ & 0.624 & & $0.000^{*}$ & 0.882 \\
\hline \multirow{3}{*}{$\begin{array}{c}\mathrm{C} \\
(\mu \mathrm{S} / \mathrm{cm})\end{array}$} & $\mathrm{N}$ & 18 & 18 & 18 & 18 & 18 & \multirow[t]{3}{*}{ 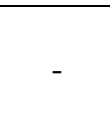 } & 18 \\
\hline & $r$ & -0.103 & -0.122 & 0.088 & -0.110 & 0.738 & & -0.366 \\
\hline & $\mathrm{p}$ & 0.684 & 0.630 & 0.728 & 0.664 & $0.000^{*}$ & & 0.135 \\
\hline \multirow{3}{*}{$\begin{array}{c}\text { Number } \\
\text { of } \\
\text { species }\end{array}$} & $\mathrm{N}$ & 18 & 18 & 18 & 18 & 18 & 18 & \multirow{3}{*}{ - } \\
\hline & $\mathrm{r}$ & -0.172 & 0.040 & -0.371 & 0.435 & -0.038 & -0.366 & \\
\hline & $\mathrm{p}$ & 0.495 & 0.876 & 0.130 & 0.071 & 0.882 & 0.135 & \\
\hline
\end{tabular}

Note:T:Temperature, DO:Dissolved oxygen, TDS: Total dissolved solids, C: Conductivity, N: Number of studied lake, r: Pearson correlation coefficient, P: Significance. ${ }^{*} \mathrm{P}<0.01$

\subsection{Diatom Communities}

A total of 95 diatom species have been recorded from epipelic, epilithic and epiphytic samples of the studied waters in the Artabel Lakes Nature Park (Table 2). The diatoms found are subdivided into three classes: Bacillariophyceae, Coscinodiscophyceae and Mediophyceae. The richest class is Bacillariophyceae, which included $89.58 \%$ of the species. Observed species were classified in 46 genera. The most abundant genera were Pinnularia (15), Eunotia (6) and Gomphonema (6); less abundant genera were Aulacoseira (4), Navicula (4), Cymbella (3), Diploneis (3), Neidium (3), and Surirella (3). Two or single species presented by other genera (Table3).

Table 2. List of the benthic diatoms in the studied lakes in the Artabel Lakes Nature Park

\begin{tabular}{|c|c|c|c|}
\hline TAXA & $\mathbf{H}$ & $\mathbf{F}$ & LAKES \\
\hline \multicolumn{4}{|l|}{ BACILLARIOPHYTA } \\
\hline \multicolumn{4}{|l|}{ Class: Coscinodiscophyceae } \\
\hline \multicolumn{4}{|l|}{ Order: Aulacoseirales } \\
\hline \multicolumn{4}{|l|}{ Family: Aulacoseiraceae } \\
\hline \multicolumn{4}{|l|}{ Genus: Aulacoseira } \\
\hline $\begin{array}{c}\text { *Aulacoseira lacustris f. tenuior Houk, Klee \& } \\
\text { Passauer }\end{array}$ & 1 & VR & ARL3 \\
\hline A. muzzanensis (F.Meister) Krammer & 1,3 & VR & ARL3,BL3 \\
\hline A. subarctica (Otto Müller) E.Y.Haworth & 1,3 & VR & ARL1 \\
\hline A. valida (Grunow) Krammer & $1,2,3$ & VR & BL4,IL \\
\hline \multicolumn{4}{|l|}{ Subclass: Melosirophycidae } \\
\hline \multicolumn{4}{|l|}{ Order: Melosirales } \\
\hline \multicolumn{4}{|l|}{ Family: Melosiraceae } \\
\hline \multicolumn{4}{|l|}{ Genus: Melosira } \\
\hline Melosira varians C.Agardh & 3 & VR & ARL3 \\
\hline \multicolumn{4}{|l|}{ Family: Orthoseiraceae } \\
\hline \multicolumn{4}{|l|}{ Genus: Orthoseira } \\
\hline $\begin{array}{l}\text { Orthoseira dendroteres (Ehrenberg) Genkal \& } \\
\text { Kulikovskiy }\end{array}$ & $1,2,3$ & $\mathrm{R}$ & ARL5,ACL3,YL2,YL3,IL \\
\hline *O. roeseana (Rabenhorst) Pfitzer & 1 & VR & YL3 \\
\hline \multicolumn{4}{|l|}{ Class: Mediophyceae } \\
\hline \multicolumn{4}{|l|}{ Subclass: Thalassiosirophycidae } \\
\hline \multicolumn{4}{|l|}{ Order: Stephanodiscales } \\
\hline \multicolumn{4}{|l|}{ Family: Stephanodiscaceae } \\
\hline \multicolumn{4}{|l|}{ Genus: Cyclotella } \\
\hline *Cyclotella ambigua Grunow & 1 & VR & ARL4 \\
\hline \multicolumn{4}{|l|}{ Genus: Discotella } \\
\hline $\begin{array}{l}\text { Discostella pseudostelligera (Hustedt) Houk \& } \\
\text { Klee }\end{array}$ & 1 & VR & ACL2 \\
\hline D. stelligera (Cleve \& Grunow) Houk \& Klee & 1 & VR & ACL3 \\
\hline \multicolumn{4}{|l|}{ Class: Bacillariophyceae } \\
\hline \multicolumn{4}{|l|}{ Subclass: Bacillariophycidae } \\
\hline \multicolumn{4}{|l|}{ Order: Bacillariales } \\
\hline Family: Bacillariaceae & & & \\
\hline
\end{tabular}


Algal Flora and Ecology of the High Mountain Lakes in the Artabel Lakes Nature Park (Gümüşhane, Turkey), I- Bacillariophyta

\begin{tabular}{|c|c|c|c|}
\hline \multicolumn{4}{|l|}{ Genus: Hantzschia } \\
\hline Hantzschia amphioxys (Ehrenberg) Grunow & 1 & $\mathrm{R}$ & ARL5,BL1,BL3,YL2,YL3,IL \\
\hline \multicolumn{4}{|l|}{ Genus: Nitzschia } \\
\hline Nitzschia perminuta (Grunow) M.Peragallo & 1 & VR & IL, YLP \\
\hline \multicolumn{4}{|l|}{ Order: Cocconeidales } \\
\hline \multicolumn{4}{|l|}{ Family: Achnanthidiaceae } \\
\hline \multicolumn{4}{|l|}{ Genus: Achnanthidium } \\
\hline Achnanthidium minutissimum (Kützing) Czarnecki & 1 & VR & BL3,IL \\
\hline \multicolumn{4}{|l|}{ Genus: Planothidium } \\
\hline $\begin{array}{l}\text { *Planothidium distinctum (Messikommer) Lange- } \\
\text { Bertalot }\end{array}$ & 1 & VR & BL4 \\
\hline $\begin{array}{l}\text { P. lanceolatum (Brébisson ex Kützing) Lange- } \\
\text { Bertalot }\end{array}$ & 1 & VR & YL2 \\
\hline \multicolumn{4}{|l|}{ Genus: Psammothidium } \\
\hline $\begin{array}{c}\text { *Psammothidium helveticum (Hustedt) } \\
\text { Bukhtiyarova \& Round }\end{array}$ & 1 & VR & ARL5 \\
\hline \multicolumn{4}{|l|}{ Family: Cocconeidaceae } \\
\hline \multicolumn{4}{|l|}{ Genus: Cocconeis } \\
\hline $\begin{array}{c}\text { Cocconeis placentula var. euglypta (Ehrenberg) } \\
\text { Grunow }\end{array}$ & $1,2,3$ & $\mathrm{R}$ & BL3,ACL2,ACL3,YL1,YLP \\
\hline \multicolumn{4}{|l|}{ Order: Cymbellales } \\
\hline \multicolumn{4}{|l|}{ Family: Cymbellaceae } \\
\hline \multicolumn{4}{|l|}{ Genus: Cymbella } \\
\hline Cymbella affinis Kützing & $1,2,3$ & $\mathrm{R}$ & ARL1,ARL2,ARL3,ARL6,BL2 \\
\hline C. aspera (Ehrenberg) Cleve & $1,2,3$ & $\mathrm{C}$ & $\begin{array}{c}\text { BL2,BL3,ACL1,ACL2,ACL3,YL1,YL } \\
\text { 2,YL3 }\end{array}$ \\
\hline C. cistula (Ehrenberg) O.Kirchner & $1,2,3$ & $\mathrm{C}$ & $\begin{array}{c}\text { ARL5,BL2,BL3,BL4,ACL1,ACL2,AC } \\
\text { L3,YL2 }\end{array}$ \\
\hline \multicolumn{4}{|l|}{ Genus: Cymbopleura } \\
\hline $\begin{array}{c}\text { Cymbopleura naviculiformis (Auerswald ex } \\
\text { Heiberg) Krammer }\end{array}$ & $1,2,3$ & $\mathrm{R}$ & ACL1,ACL2,ACL3,YL2,IL \\
\hline C. subcuspidata (Krammer) Krammer & 1 & VR & ARL6 \\
\hline \multicolumn{4}{|l|}{ Genus: Didymosphenia } \\
\hline $\begin{array}{c}\text { Didymosphenia geminata (Lyngbye) } \\
\text { Mart.Schmidt }\end{array}$ & $1,2,3$ & $\mathrm{C}$ & $\begin{array}{l}\text { ARL5,BL1,BL2,BL3,ACL1,ACL2,AC } \\
\text { L3,YL1,YL3,YLP }\end{array}$ \\
\hline \multicolumn{4}{|l|}{ Family: Gomphonemataceae } \\
\hline \multicolumn{4}{|l|}{ Genus: Encyonema } \\
\hline Encyonema gracile Rabenhorst & 3 & VR & ARL1,YL2 \\
\hline E. minutum (Hilse) D.G.Mann & $1,2,3$ & $\mathrm{~F}$ & $\begin{array}{c}\text { ARL5,ARL6,BL1,BL2,BL3,BL4,ACL } \\
\text { 1,ACL2,ACL3,YL1,YL2,YL3,IL, } \\
\text { YLP }\end{array}$ \\
\hline \multicolumn{4}{|l|}{ Genus: Gomphonema } \\
\hline Gomphonema acuminatum Ehrenberg & 1,3 & VR & ACL2,ACL3 \\
\hline G. angustatum (Kützing) Rabenhorst & 1,3 & VR & YL2,YL3,IL \\
\hline G. augur Ehrenberg & $1,2,3$ & VR & ACL2,ACL3 \\
\hline G. calcareum Cleve & 1 & VR & IL,YLP \\
\hline G. olivaceum (Hornemann) Brébisson & $1,2,3$ & $\mathrm{C}$ & $\begin{array}{c}\text { BL2,BL3,ACL2,YL1,YL2,YL3,IL,YL } \\
\text { P }\end{array}$ \\
\hline G. parvulum (Kützing) Kützing & $1,2,3$ & $\mathrm{R}$ & ARL1,ARL5,ARL6,BL3,BL4,YL3,IL \\
\hline \multicolumn{4}{|l|}{ Genus: Placoneis } \\
\hline Placoneis hambergii (Hustedt) K.Bruder & 1 & VR & ACL1 \\
\hline \multicolumn{4}{|l|}{ Order: Mastogloiales } \\
\hline \multicolumn{4}{|l|}{ Family: Achnanthaceae } \\
\hline \multicolumn{4}{|l|}{ Genus: Achnanthes } \\
\hline Achnanthes semiaperta Hustedt & 1 & VR & ARL5,BL4 \\
\hline \multicolumn{4}{|l|}{ Genus: Platessa } \\
\hline Platessa salinarum (Grunow) Lange-Bertalot & 1 & VR & ARL5 \\
\hline \multicolumn{4}{|l|}{ Order: Naviculales } \\
\hline Suborder: Diploneidineae & & & \\
\hline Family: Diploneidaceae & & & \\
\hline
\end{tabular}


Algal Flora and Ecology of the High Mountain Lakes in the Artabel Lakes Nature Park (Gümüşhane, Turkey), I- Bacillariophyta

\begin{tabular}{|c|c|c|c|}
\hline Genus: Diploneis & & & \\
\hline $\begin{array}{l}\text { Diploneis oblongella (Nägeli ex Kützing) Cleve- } \\
\text { Euler }\end{array}$ & 1,3 & VR & ARL5,ACL2 \\
\hline D. parma Cleve & 3 & VR & YLP \\
\hline$* D$. petersenii Hustedt & 3 & VR & YLP \\
\hline \multicolumn{4}{|l|}{ Suborder: Naviculineae } \\
\hline \multicolumn{4}{|l|}{ Family: Naviculaceae } \\
\hline \multicolumn{4}{|l|}{ Genus: Caloneis } \\
\hline Caloneis alpestris (Grunow) Cleve & 1 & VR & ARL4,ACL1 \\
\hline C. silicula (Ehrenberg) Cleve & 1,2 & $\mathrm{C}$ & $\begin{array}{l}\text { ARL5,ARL6,BL1,BL2,BL3,ACL1,AC } \\
\text { L2,ACL3,YL1 }\end{array}$ \\
\hline \multicolumn{4}{|l|}{ Genus: Chamaepinnularia } \\
\hline $\begin{array}{c}\text { Chamaepinnularia hassiaca (Krasske) Cantonati } \\
\text { \& Lange-Bertalot }\end{array}$ & 1 & VR & IL \\
\hline \multicolumn{4}{|l|}{ Genus: Mayamaea } \\
\hline Mayamaea atomus (Kützing) Lange-Bertalot & 1 & VR & IL \\
\hline \multicolumn{4}{|l|}{ Genus: Navicula } \\
\hline Navicula cryptocephala Kützing & 1,3 & $\mathrm{R}$ & BL3,ACL1,ACL2,ACL3,YL1,YL2,IL \\
\hline N. radiosa Kützing & 1,3 & $\mathrm{R}$ & $\begin{array}{l}\text { ARL3,ARL5,ARL6,BL1,BL3,BL4,YL } \\
3\end{array}$ \\
\hline N. rhynchocephala Kützing & 1 & $\mathrm{R}$ & BL3,ACL2,YL3,IL \\
\hline N. veneta Kützing & 1 & VR & ACL1,YLP \\
\hline \multicolumn{4}{|l|}{ Family: Stauroneidaceae } \\
\hline \multicolumn{4}{|l|}{ Genus: Craticula } \\
\hline Craticula cuspidata (Kützing) D.G.Mann & 1 & $\mathrm{R}$ & ARL4,ARL5,BL4,ACL2,YL1 \\
\hline C. halophilioides (Hustedt) Lange-Bertalot & 1,3 & VR & ARL1,IL \\
\hline \multicolumn{4}{|l|}{ Genus: Stauroneis } \\
\hline Stauroneis acuta W.Smith & 2 & VR & ACL1 \\
\hline S. anceps Ehrenberg & 1,3 & $\mathrm{~F}$ & $\begin{array}{l}\text { ARL1,ARL3,ARL4,ARL5,ARL6,BL3, } \\
\text { BL4,ACL2,ACL3,YL2,YL3,IL }\end{array}$ \\
\hline \multicolumn{4}{|l|}{ Suborder: Neidiineae } \\
\hline \multicolumn{4}{|l|}{ Family: Amphipleuraceae } \\
\hline \multicolumn{4}{|l|}{ Genus: Frustulia } \\
\hline $\begin{array}{c}\text { *Frustulia crassinervia (Brébisson ex W.Smith) } \\
\text { Lange-Bertalot \& Krammer }\end{array}$ & 1,3 & $\mathrm{R}$ & ARL1,ARL4,BL1,BL4,YL2,YLP \\
\hline F. vulgaris (Thwaites) De Toni & 2 & VR & BL2 \\
\hline \multicolumn{4}{|l|}{ Genus: Halamphora } \\
\hline Halamphora coffeiformis (C.Agardh) Levkov & 1,2 & VR & BL4,ACL1 \\
\hline \multicolumn{4}{|l|}{ Family: Neidiaceae } \\
\hline \multicolumn{4}{|l|}{ Genus: Neidium } \\
\hline Neidium affine (Ehrenberg) Pfitzer & 1 & VR & ARL4 \\
\hline N. ampliatum (Ehrenberg) Krammer & $1,2,3$ & $\mathrm{R}$ & ACL2,ACL3,YL2,IL \\
\hline N. iridis (Ehrenberg) Cleve & 1,3 & $\mathrm{R}$ & ARL3,ARL5,BL1,BL3,ACL3,IL \\
\hline \multicolumn{4}{|l|}{ Genus: Neidiomorpha } \\
\hline $\begin{array}{c}\text { Neidiomorpha binodis (Ehrenberg) M.Cantonati, } \\
\text { Lange-Bertalot \& N.Angeli }\end{array}$ & 1 & VR & ARL6 \\
\hline \multicolumn{4}{|l|}{ Suborder: Sellaphorineae } \\
\hline \multicolumn{4}{|l|}{ Family: Pinnulariaceae } \\
\hline \multicolumn{4}{|l|}{ Genus: Pinnularia } \\
\hline Pinnularia abaujensis (Pantocsek) R.Ross & 1 & VR & BL3 \\
\hline P. aestuarii Cleve & 1,3 & $\mathrm{R}$ & BL1,YL2,YL3,YLP \\
\hline P. borealis Ehrenberg & $1,2,3$ & $\mathrm{~F}$ & $\begin{array}{c}\text { ARL1,ARL2,ARL3,ARL5,ARL6,BL1, } \\
\text { BL4,ACL1,ACL3,YL1,YL2,YL3, } \\
\text { IL,YLP }\end{array}$ \\
\hline P. divergens W.Smith & 1 & VR & ACL3 \\
\hline P. episcopalis Cleve & 1,3 & VR & IL \\
\hline P. hemiptera (Kützing) Rabenhorst & 1 & VR & ARL4,BL1 \\
\hline P. interrupta W.Smith & $1,2,3$ & VF & $\begin{array}{l}\text { ARL1,ARL2,ARL3,ARL4,ARL5,ARL } \\
\text { 6,BL1,BL3,BL4,ACL1,ACL2, }\end{array}$ \\
\hline
\end{tabular}


Algal Flora and Ecology of the High Mountain Lakes in the Artabel Lakes Nature Park (Gümüşhane, Turkey), I- Bacillariophyta

\begin{tabular}{|c|c|c|c|}
\hline & & & ACL3,YL1,YL2,YL3,IL,YLP \\
\hline P. lata (Brébisson) W.Smith & 1 & VR & BL4 \\
\hline P. major (Kützing) Rabenhorst & 1,3 & $\mathrm{~F}$ & $\begin{array}{l}\text { ARL1,ARL3,ARL5,ARL6,BL1,BL4,A } \\
\text { CL1,ACL3,YL2,YL3,IL,YLP }\end{array}$ \\
\hline P. mesogongyla Ehrenberg & 1,3 & VR & ARL1,BL1 \\
\hline P. obscura Krasske & 1 & VR & ARL5 \\
\hline P. rupestris Hantzsch & 1 & VR & ARL3 \\
\hline P. subcapitata W.Gregory & 1 & $\mathrm{R}$ & ARL1,ARL2,ARL4,ARL5,YLP \\
\hline P. viridiformis Krammer & 1 & VR & YL2 \\
\hline P. viridis (Nitzsch) Ehrenberg & $1,2,3$ & $\mathrm{R}$ & ARL1,ARL2,ARL3,BL1,YL2,YL3,IL \\
\hline \multicolumn{4}{|l|}{ Family: Sellaphoraceae } \\
\hline \multicolumn{4}{|l|}{ Genus: Sellaphora } \\
\hline Sellaphora pupula (Kützing) Mereschkovsky & 1,2 & VR & BL1,ACL1 \\
\hline \multicolumn{4}{|l|}{ Order: Rhopalodiaceae } \\
\hline \multicolumn{4}{|l|}{ Family: Rhopalodiaceae } \\
\hline \multicolumn{4}{|l|}{ Genus: Epithemia } \\
\hline Epithemia gibba (Ehrenberg) Kützing & $1,2,3$ & $\mathrm{C}$ & $\begin{array}{c}\text { ARL1,ARL3,ARL4,ARL6,ACL2,ACL } \\
\text { 3,YL2,YL3,IL,YLP }\end{array}$ \\
\hline \multicolumn{4}{|l|}{ Order: Surirellales } \\
\hline \multicolumn{4}{|l|}{ Family: Surirellaceae } \\
\hline \multicolumn{4}{|l|}{ Genus: Iconella } \\
\hline Iconella linearis (W.Smith) Ruck \& Nakov & $1,2,3$ & VR & ARL5,IL,YLP \\
\hline \multicolumn{4}{|l|}{$\begin{array}{c}\text { Genus: Surirella } \\
\end{array}$} \\
\hline Surirella angusta Kützing & 1,2 & $\mathrm{C}$ & $\begin{array}{c}\text { ARL1,ARL5,ARL6,BL1,BL4,ACL2,Y } \\
\text { L1,YL3,IL }\end{array}$ \\
\hline S. robusta Ehrenberg & 1 & $\mathrm{R}$ & ARL4,ARL5,ARL6,ACL1,YL3,IL \\
\hline S. splendida (Ehrenberg) Kützing & $1,2,3$ & $\mathrm{~F}$ & $\begin{array}{c}\text { ARL1,ARL4,ARL5,ARL6,BL1,BL3,B } \\
\text { L4,ACL1,ACL2,ACL3,YL1,YL2,YL3, } \\
\text { YLP }\end{array}$ \\
\hline \multicolumn{4}{|l|}{ Order: Thalassiophysales } \\
\hline \multicolumn{4}{|l|}{ Family: Catenulaceae } \\
\hline \multicolumn{4}{|l|}{ Genus: Amphora } \\
\hline Amphora ovalis (Kützing) Kützing & $1,2,3$ & $\mathrm{~F}$ & $\begin{array}{l}\text { ARL5,ARL6,BL1,BL3,BL4,ACL1,AC } \\
\text { L2,ACL3,YL1,YL2,IL,YLP }\end{array}$ \\
\hline A. pediculus (Kützing) Grunow & 3 & VR & YL3 \\
\hline \multicolumn{4}{|l|}{ Subclass: Eunotiophycidae } \\
\hline \multicolumn{4}{|l|}{ Order: Eunotiales } \\
\hline \multicolumn{4}{|l|}{ Family: Eunotiaceae } \\
\hline \multicolumn{4}{|l|}{ Genus: Eunotia } \\
\hline Eunotia arcus Ehrenberg & 1,3 & VR & ARL3,YL3 \\
\hline E. bilunaris (Ehrenberg) Schaarschmidt & 1,3 & $\mathrm{R}$ & ARL3,ARL4,ARL5,BL4,IL \\
\hline E. diodon Ehrenberg & 1,3 & $\mathrm{C}$ & $\begin{array}{l}\text { ARL2,ARL4,ARL5,BL1,BL4,YL1,YL } \\
\text { 2,IL }\end{array}$ \\
\hline $\begin{array}{l}\text { *E. mucophila (Lange-Bertalot, Nörpel-Schempp } \\
\text { \& Alles) Lange- Bertalot } \\
\end{array}$ & $1,2,3$ & $\mathrm{R}$ & ARL1,ARL3,ACL2,YL2,IL \\
\hline *E. paludosa Grunow & 2 & VR & $\mathrm{IL}$ \\
\hline E. praerupta Ehrenberg & $1,2,3$ & $\mathrm{VF}$ & $\begin{array}{l}\text { ARL1,ARL2,ARL3,ARL4,ARL5,BL2, } \\
\text { BL3,BL4,ACL2,ACL3, YL1, YL2,YL3, } \\
\text { IL,YLP }\end{array}$ \\
\hline \multicolumn{4}{|l|}{ Subclass: Fragilariophycidae } \\
\hline \multicolumn{4}{|l|}{ Order: Fragilariales } \\
\hline \multicolumn{4}{|l|}{ Family: Fragilariaceae } \\
\hline \multicolumn{4}{|l|}{ Genus: Odontidium } \\
\hline Odontidium mesodon (Kützing) Kützing & $1,2,3$ & $\mathrm{R}$ & ARL1,ACL1,ACL2,YL2 \\
\hline \multicolumn{4}{|l|}{ Family: Staurosiraceae } \\
\hline \multicolumn{4}{|l|}{ Genus: Staurosira } \\
\hline Staurosira venter (Ehrenberg) Cleve \& J.D.Möller & 1 & VR & BL5,IL \\
\hline \multicolumn{4}{|l|}{$\begin{array}{l}\text { Genus: Staurosirella } \\
\end{array}$} \\
\hline $\begin{array}{c}\text { Staurosirella pinnata (Ehrenberg) D.M.Williams } \\
\text { \& Round }\end{array}$ & 1 & VR & $\mathrm{IL}$ \\
\hline
\end{tabular}


Algal Flora and Ecology of the High Mountain Lakes in the Artabel Lakes Nature Park (Gümüşhane, Turkey), I- Bacillariophyta

\begin{tabular}{|c|c|c|c|}
\hline Order: Licmophorales & & & \\
\hline \multicolumn{4}{|l|}{ Family: Ulnariaceae } \\
\hline \multicolumn{4}{|l|}{ Genus: Hannaea } \\
\hline Hannaea arcus (Ehrenberg) R.M.Patrick & $1,2,3$ & $\mathrm{C}$ & $\begin{array}{c}\text { ARL5,ARL6,BL2,BL3,ACL1,ACL2,A } \\
\text { CL3,YL1 }\end{array}$ \\
\hline \multicolumn{4}{|l|}{ Genus: Ulnaria } \\
\hline Ulnaria ulna (Nitzsch) Compère & $1,2,3$ & $\mathrm{R}$ & ARL5,BL2,BL3,YL3 \\
\hline \multicolumn{4}{|l|}{ Order: Tabellariales } \\
\hline \multicolumn{4}{|l|}{ Family: Tabellariaceae } \\
\hline \multicolumn{4}{|l|}{ Genus: Diatoma } \\
\hline Diatoma vulgaris Bory & 1,2 & $\mathrm{C}$ & $\begin{array}{c}\text { ARL5,ARL6,BL1,BL2,BL3,BL4,ACL } \\
\text { 1,ACL2,IL }\end{array}$ \\
\hline \multicolumn{4}{|l|}{ Genus: Fragilaria } \\
\hline Fragilaria crotonensis Kitton & 2 & VR & ACL2 \\
\hline $\begin{array}{c}\text { F. tenera var. nanana (Lange-Betalot) Lange- } \\
\text { Bertalot \& S.Ulrich }\end{array}$ & $1,2,3$ & VR & ACL1,ACL2,ACL3 \\
\hline \multicolumn{4}{|l|}{ Genus: Meridion } \\
\hline Meridion circulare (Greville) C.Agardh & $1,2,3$ & $\mathrm{C}$ & $\begin{array}{c}\text { ARL1,ARL5,BL1,BL2,BL3,ACL3,YL } \\
\text { 1,YL2,YL3,YLP }\end{array}$ \\
\hline \multicolumn{4}{|l|}{ Genus: Tabellaria } \\
\hline Tabellaria flocculosa (Roth) Kützing & $1,2,3$ & $\mathrm{~F}$ & $\begin{array}{c}\text { ARL1,ARL3,ARL4,ARL5,ARL6,BL2, } \\
\text { BL3,BL4,ACL2,ACL3,IL }\end{array}$ \\
\hline
\end{tabular}

Note: H: Habitat; 1: Epipelic, 2: Epilithic, 3: Epiphytic; F: Frequences; ARL: Artabel Lakes, BL: Beş Lakes, YL: Yıldız Lakes, ACL: Acembol Lakes, IL: Issimsiz Lake, YLP: Yıldı Lakes Pond; *: New record for diatom flora of Turkey.

Table 3. List of the diatom genera with the number of species

\begin{tabular}{|c|c|c|c|}
\hline Genus & Number of species & Genus & Number of species \\
\hline Achnanthes & 1 & Hantzschia & 1 \\
\hline Achnanthidium & 1 & Iconella & 1 \\
\hline Amphora & 2 & Melosira & 1 \\
\hline Aulacoseira & 4 & Meridion & 1 \\
\hline Caloneis & 2 & Mayamaea & 1 \\
\hline Chamaepinnularia & 1 & Navicula & 4 \\
\hline Cocconeis & 1 & Neidiomorpha & 1 \\
\hline Craticula & 2 & Neidium & 3 \\
\hline Cyclotella & 1 & Nitzschia & 1 \\
\hline Cymbella & 3 & Odontidium & 1 \\
\hline Cymbopleura & 2 & Orthoseria & 2 \\
\hline Diatoma & 1 & Pinnularia & 15 \\
\hline Didymosphenia & 1 & Placoneis & 1 \\
\hline Diploneis & 3 & Planothidium & 2 \\
\hline Discotella & 2 & Platessa & 1 \\
\hline Encyonema & 2 & Psammothidium & 1 \\
\hline Epithemia & 1 & Sellaphora & 1 \\
\hline Eunotia & 6 & Stauroneis & 2 \\
\hline Fragilaria & 2 & Staurosira & 1 \\
\hline Frustulia & 2 & Staurosirella & 1 \\
\hline Gomphonema & 6 & Surirella & 3 \\
\hline Halamphora & 1 & Tabellaria & 1 \\
\hline Hannaea & 1 & Ulnaria & 1 \\
\hline
\end{tabular}

On the base of revealed diatom species list, we constructed the Willis curve to assess how representative the diatoms were in the studied lakes. The Willis curve of the diatom flora in the Artabel Lakes Nature Park was determined to be close to the hyperbolic shape (Fig. 3). This situation shows that we have sufficient diatom species in order to make taxonomic and ecological analysis. Earlier, S. Barinova [24] found that the Willis curve can be used as criteria for fullness of species list because the distribution follows the hyperbolic shape in well-studied algal floras in Eurasia. Therefore, we can do floristic, taxonomic and ecological analysis for the diatom flora in the Artabel 
Algal Flora and Ecology of the High Mountain Lakes in the Artabel Lakes Nature Park (Gümüşhane, Turkey), I- Bacillariophyta

Lakes Nature Park and compare it to other high mountain lake floras in this region where the Willis proportion also closely follows the hyperbolic shape [24-28].

\section{Willis curve}

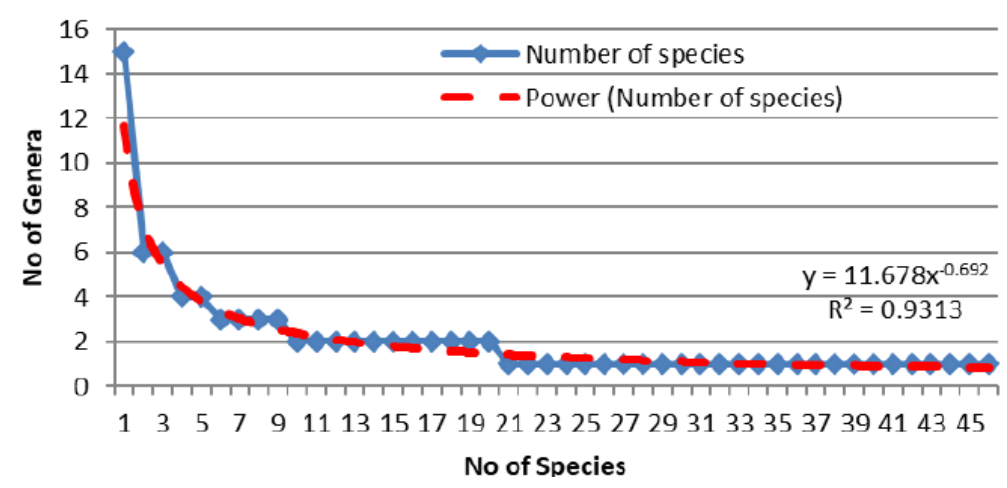

Figure 3. The Willis curve for the diatom flora of the Artabel Lakes Nature Park shows the number of species per genera (i.e., 1 genus with 15 species, 2 genera with 6 species, etc.).

One of the important characteristics of the Artabel Lakes Nature Park diatom flora is the composition of the dominant algae genera. Only three genera (Pinnularia, Eunotia and Gomphonema) contain $28.42 \%$ of the diatom flora in the studied waters (Table 3). However, two genera of the three are usually in the head part of the diatom flora list, surprisingly, the members of Pinnularia have been influential in the diatom flora of the Artabel Lakes Nature Park. Another important characteristic of the flora is that 9 new records diatom species have been detected for Turkey (Table 2). Information on descriptions, ecology and geographic distribution of these species were published in a separate paper [29].

Only Eunotia praerupta and Pinnularia interrupta are considered very frequent (VF) (2.10\%) species. They are identified in more than 15 of the lakes examined. Frequently $(\mathrm{F})$ recorded diatom species (Amphora ovalis, Encyonema minutum, Pinnularia borealis, P. major, Stauroneis anceps, Surirella splendia and Tabellaria flocculosa) comprise $7.36 \%$ of all diatom flora. Common (C) species which are Caloneis silicula, Cymbella aspera, C. cistula, Diatoma vulgaris, Didymosphenia geminata, Epithemia gibba, Eunotia diodon, Gomphonema olivaceum, Hannaea arcus, Meridion circulare, and Surirella angusta, comprise $11.57 \%$ of all diatom species encountered. Remarkably, $78.94 \%$ of the diatom species reported as rare (R) or very rare (VR) species (Table 2).

The genus Pinnularia was represented by 15 species and accounted for $15.78 \%$ of the diatom flora of the studied waters (Table 3). A similar situation was observed in the diatom flora of the Pamir area in Tajikistan [28]. Wehr and Sheath [30] reported that the members of Pinnularia is often abundant in low conductivity and slightly acidic freshwater. The results of this study showed that the waters are mostly circumneutral and acidic and have low mineral content [23]. These ecological conditions of the waters formed suitable environments for the development of the genus Pinnularia. Pinnularia interrupta is the first important species of the diatom flora; it was found in 17 sites and recorded as very frequent (VF) (94.44\%) (Table 2). Patrick and Reimer [11] state that Pinnularia interrupta prefers low mineral content freshwater. According to Van Dam et al., [31] it occurs in circumneutral, oligosaprobic, and oligo-mesotrophic waters. Pinnularia borealis was found in all benthic habitats and recorded as frequent (F) (77.77\%) (Table 2). According to Patrick and Reimer [11], the species prefers cool waters of low mineral content and is often found in rivers, but also in ponds. It also prefers circumneutral, $\beta$-mesosaprobic and oligo-mesotrophic waters [31]. Pinularia major is widely found in waters of fairly low mineral content [11]. It also occurs in circumneutral, $\beta$-mesosaprobic and meso-eutrophic waters [31]. This species was found in the epipelic and epiphytic samples and is considered as frequent (F) 66.66\% (Table 2).

Krammer and Lange-Bertalot [13], Wojtal et al. [32] and Hofmann et al. [33] stated that Eunotia species were present in tested waters, which have low-to-moderate concentrations of electrolytes, and have $\mathrm{pH}$ ranges from acidic to neutral. While the water temperature does not have a significant effect in the widespread species of this genus, the $\mathrm{pH}$ and electrolyte content are important factors [34]. The genus Eunotia has a wide distribution in the high mountain lakes in Turkey [35-37]. Because they provide the suitable conditions which are saying above for the Eunotia genus distribution. In this 
investigation, physico-chemical analysis of the waters showed that the $\mathrm{pH}$ was between 6.16-7.52 and conductivity was $12.0-49.9 \mu \mathrm{S} / \mathrm{cm}$, which confirms conditions for the presence of the genus Eunotia [23], which was represented by 6 species in the diatom flora of the Artabel Lakes Nature Park (Table 4). Eunotia praerupta is the second important species of the diatom flora; it was found in 15 sites and recorded as very frequent (VF) (83.33\%) (Table 2). According to Patrick and Reimer [11], this species occurs usually in northern or mountainous areas in acid to circumneutral waters. It is acidophilus and mainly occurring at $\mathrm{pH}<7$ [31]. Eunotia diodon is a cosmopolitan species. It is often found in northern Europe, as well as in the waters of high mountain regions of Central Europe. These waters are characterized by low-to-moderate conductivity [13]. It is also common in waters with $\mathrm{pH}<7$ [31]. According to Krammer and Lange-Bertalot [13] and Wojtal et al. [32], this species is found on wet rocks, between mosses, and in mud substrate. In this study, it was found in the epipelic and epiphytic samples, recorded as common (C) (44.44\%) (Table 2). Patrick and Reimer [11] noted that Eunotia bilunaris is widespread in low mineral content waters. It is commonly found in acid waters, but may also occur in slightly alkaline waters. Alles et al. [38] suggest that this taxon has two optima in terms of $\mathrm{pH}$ values. The first one is around 4.0-4.3, the second one is $\mathrm{pH} 7$ or slightly above. According to Van Dam et al [31], this species has no apparent optimum in $\mathrm{pH}$. In the studied waters, this species was collected in epipelic and epiphytic habitats with $\mathrm{pH}$ of 6.19-7.04, conductivity of $12-49.9 \mu \mathrm{S} / \mathrm{cm}$, and an altitude of $2668-2930 \mathrm{~m}$ a.s.l. It was recorded as rare (R) $(27.77 \%)$ (Table 2). In addition, Eunotia mucophila and E. paludosa have been identified as new records for the Freshwater Algal Flora of Turkey.

According to Wehr and Sheath [30], the genus Gomphonema is found in nearly every habitat type within circumneutral lakes and streams. Although the genus Gomphonema represented by six species in the diatom flora, only Gomphonema olivaceum was recorded as common (C) (44.44\%) (Table 2). The others were recorded as rare and very rare (Table 2). This species seems to prefer cool, flowing water, which is fairly hard, although it is found in water with great variation in its calcium content. It does not seem to thrive in water with a very low calcium content [11]. Van Dam et al. [31] state that this species is alkalibiontic, $\beta$-mesosaprobic and eutrophic.

The members of the genus Surirella are benthic and are found most often on epipelic habitats but may also occupy the epilithic and epiphytic [30]. The only species that received attention in this genus is Surirella splendida. It was found in the epipelic, epilithic and epiphytic samples of the 14 sites and recorded as frequent (F) (77.77\%) (Table 2). This species has been reported from alkaliphilous (mainly occuring at $\mathrm{pH}>7$ ), $\beta$-mesosaprobous and meso-eutrophic waters [31]. According to Krammer and Lange-Bertalot [15], Surirella splendida prefers eutrophic waters with moderate-to- high electrolyte content and is also found in various salt mines, but less common in Alpine lakes.

According to literature, Amphora ovalis seems to be characteristic of standing or slowly flowing waters [11], is alkaliphilic, $\beta$-mesosaprobic, and eutrophic [31]. In this study, it was found in circumneutral, acidophilous and low- mineral content waters (Fig. 2) and recorded as frequent (F) (66.66\%) (Table 2). Encyonema minutum, which was recorded as frequent (F) (77.77\%) (Table 2), has a wide geographical distribution [11] and prefers circumneural waters [31]. Stauroneis anceps, which was found in the twelve sites, is one of the frequent $(\mathrm{F})(66.66 \%)$ species in the diatom flora (Table 2 ). This species prefers circumneutral, $\beta$-mesosaprobic, and meso-eutrophic waters [31]. According to Patrick and Reimer [11], this species has a wide geographical distribution and prefers $\mathrm{pH}$ indifferent waters. Tabellaria flocculosa is recorded as frequent (F) (61.11\%) (Table 2). This species has a wide tolerance for different types of water. Tabellaria flocculosa, which has shorter frustules, more often occur in acid waters of bogs and ponds; however, those with longer frustules were found in oligotrophic and mesotrophic waters [11]. Van Dam et al. [31] state that it prefers acidophilic, $\beta$ mesosaprobic, and mesotrophic water. One of the most commonly found benthic algae samples in all freshwater ecosystems is also Achnanthidium minutissimum [11, 14]. Van Dam et al [31] state that this species had been found in acidic and alkaline, oligotrophic and hypertrophic waters. Whereas, in this study, this species was found only in the epipelic samples of the two lakes (BL3 and IL) and recorded as very rare (VR) (11.11\%) (Table 2).

The investigation of the taxonomic structure of the flora is an important part of the floristic analysis, which makes it possible to identify the most diversified species of the floristic spectrum, showing the direction of the algal flora development of the region as a whole [28]. In conclusion, the diatom flora 
Algal Flora and Ecology of the High Mountain Lakes in the Artabel Lakes Nature Park (Gümüşhane, Turkey), I- Bacillariophyta

of the Artabel Lakes Nature Park was formed by cosmopolitan species with a large involvement of species from alpine and subalpine area, as was found in the other studied high mountain lakes in the region [35-37,39,40,41,42,43,44]. When the benthic habitats of the studied lakes were compared, differences were observed in species composition and relative abundance of each lake. The diatom flora of Artabel Lakes Nature Park is composed of indifferent (48.31\%), alkaliphiles (31.46\%), and acidophiles $(16.85 \%)$ species. It was also identified north-alpine and alpine diatom species. The physico-chemical conditions of the studied waters also provided a suitable environment for the development of the diatom flora. In this study, a total of 95 species belonging to Bacillariophyta were identified only in benthic habitats. Whereas, Atıc1 [6] recorded 58 diatom species in the plankton and benthic habitats of the 18 lakes in the Artabel Lakes Nature Park. These findings highlighted the necessity for further fundamental investigations in the Artabel Lakes Nature Park. The data obtained from this study contribute to information about both bioindicator diatom species and high mountain lakes.

\section{ACKNOWLEDGMENT}

This project was financially supported by Karadeniz Technical University (BAP: FBA-2016-5487). We are grateful to teacher Vehbi YALÇIN for his support in the field. The work of the third author was partly supported by the Israeli Ministry of Aliyah an Integration.

\section{REFERENCES}

[1] Biggs B. J. F., Patterns in periphyton of streams. In R. J. Stevenson, M. L. Bothwell, R. L. Lowe, editors. Algal ecology: Freshwater benthic ecosystems, Academic, 1996.

[2] Ács É., Szabó K., Tóth B and Kiss K. T., Investigation of benthic algal communities, especially diatoms of some Hungarian streams in connection with reference conditions of the Water Framework Directives, Acta Botanica Hungarica, 46, 255-277, 2004.

[3] Bere T. and Tundisı J. G., Biological monitoring of lotic ecosystems: the role of diatoms, Brazilian Journal of Biology, 70, 493-502, 2010.

[4] Krizmanić J., Ilić M., Vidaković D., Subakov-Simić G., Petrović J.and Cvetanović K., Diatoms of the Dojkinci River (Stara Planina Nature Park, Serbia), Acta Botanica Croatica, 74, 317-331, 2015.

[5] Cantonati M. and Lange-Bertalot H., Diatom monitors of close-to-pristine, very-low alkalinity habitats: three new Eunotia species from springs in Nature Parks of the southeastern Alps, Journal of Limnology, 70, 209-221, 2011.

[6] Atıcı T., Use of cluster analyze and similarity of algae in Eastern Black Sea Region Glacier Lakes (Turkey), Key area: Artabel Lakes Natural Park, Gazi University Journal of Science, 31, 25-40, 2018.

[7] Doğa Koruma ve Milli Parklar Genel Müdürlüğü, Artabel Gölleri Tabiat Parkı Uzun Devreli Gelişme Planı Analitik Etüt ve Sentez Raporu. Ankara: Doğa Koruma ve Milli Parklar Genel Müdürlüğü, 2013.

[8] Battarbee R. W., Diatom analysis. In: Berglund BE editor, Handbook of Holocene palaeoecology and palaehydrology, Chicester, USA, John Wiley and Sons, 1986.

[9] Hustedt F., Die Süsswasser Flora von Mitteleuropas, Heft 10: Bacillariophyta (Diatomeae), Zweite Auflage, Germany, Jena Verlag von Gustav Fischer, 1930.

[10] Huber-Pestalozzi G., Das Phytoplankton des Süßwassers systematic und biologie (Die Binnengewässer, Band XVI), Teil 2., 2. Hälfte, Diatomeen, Stuttgart, Germany, E. Schweizerbart'sche Verlagsbuchhandlung (Nagele u. Obermiller), 1942.

[11] Patrick R. and Reimer C. W., The Diatoms of the United States, Exclusive of Alaska and Hawaii: Fragilariaceae, Eunotiaceae, Achnanthaceae, Naviculacae, Philadelphia, USA, Academy of Natural Sciences, 1966.

[12] Krammer K. and Lange-Bertalot H., Bacillariophyceae 1.Teil: Naviculaceae, Süßwasserflora von Mitteleuropa, 2 (1), Berlin, Germany, Gustav Fischer Verlag, 1986.

[13] Krammer K. and Lange-Bertalot H., Bacillariophyceae 3. Teil: Centrales, Fragilariaceae, Eunotiaceae, Süßwasserflora von Mitteleuropa, Band 2(3), Berlin, Germany, Gustav Fischer Verlag, 1991.

[14] Krammer K. and Lange-Bertalot H., Bacillariophyceae 4. Teil: Achnanthaceae, Kritische Ergänzungen zu Navicula (Lineolatae) und Gomphonema, Süßwasserflora von Mitteleuropa, Band 2 (4), Berlin, Germany, Gustav Fischer Verlag, 1991.

[15] Krammer K. and Lange-Bertalot H., Bacillariophyceae, 1. Teil: Naviculaceae, Süßwasserflora von Mitteleuropa, Band 2 (1), Berlin, Germany: Gustav Fischer Verlag, 1999. 
[16] Joh G., Algal Flora of Korea, Chrysophyta: Bacillariophyceae: Centrales Freshwater Diatoms I, Vol. 3(1), Seo-gu Incheon, Korea, National Institute of Biological Resources Ministry of Environment, 2010.

[17] Joh G., Algal Flora of Korea, Chrysophyta: Bacillariophyceae: Pennales: Raphidineae: Achnanthaceae, Freshwater Diatoms, Vol. 3(7), Seo-gu Incheon, Korea, National Institute of Biological Resources Ministry of Environment, 2012.

[18] Genkal S. I., Pautova V. N., Nomokonova V. N. and Tarasova N. G., Occurence of Cyclotella ambigua Grunow (Bacillariophyta) in the Kuibyshev Reservoir, Inland Water Biology, 1, 7-13, 2008.

[19] Buczkó K., Wojtal A. Z. and Magyari E. K., Lectotypification, emended description and distribution of Planothidium distinctum (Achnanthidiaceae, Bacillariophyceae), Phytotaxa, 117, 1-10, 2013.

[20] Jovanovska E., Levkov Z. and Edlund M. B., The genus Diploneis Ehrenberg ex Cleve (Bacillariophyta) from Lake Hövsgöl, Mongolia, Phytotaxa, 217, 201-248, 2015. http:/ /www.algaebase.org.

[21] Kocataş A., Ekoloji (Çevre Biyolojisi), İzmir, Türkiye, Ege Üniversitesi Matbaası, 1992.

[22] Şahin B. and Akar B., New records from Artabel Lakes Nature Park (Gümüşhane/Turkey) to the freshwater algal flora of Turkey, Turkish Journal of Botany, 43, 135-142, 2019.

[23] Barinova S., Systemic criteria for the analysis of alpha- and gamma-diversity of freshwater algae, International Journal of Environmental Sciences \& Natural Resources, 4, 555-633, 2017.

[24] Barinova S., Algal diversity dynamics, ecological assessment, and monitoring in the river ecosystems of the eastern Mediterranean, New York, USA, Nova Science Publishers, 2011.

[25] Barinova S., Kukhaleishvili L., Nevo E. and Janelidze Z., Diversity and ecology of algae in the Algeti National Park as a part of the Georgian system of protected areas, Turkish Journal of Botany, 35, 729-774, 2011.

[26] Barinova S., Boboev M. and Hisoriev H., Freshwater algal diversity of the South-Tajik Depression in a high mountainous extreme environment, Turkish Journal of Botany, 39, 535-546, 2015.

[27] Niyatbekov T. and Barinova S., Diatom species richness in algal flora of Pamir, Tajikistan, European Scientific Journal, 14, 301-323, 2018.

[28] Şahin B. and Akar B., Nine new records from high mountain lakes (Artabel Lakes Nature Park, Gümüşhane/Turkey) for the freshwater distom flora of Turkey, BioDiCon, 11, 56-63, 2018.

[29] Wehr J. D. and Sheath R. G.,Freshwater algae of North America (Ecology and Classification), California, USA, Academic Press, 2003.

[30] Van Dam H., Mertens A. and Sinkeldam J., A coded checklist and ecological indicator values of freshwater diatoms from the Netherlands, Netherland Journal of Aquatic Ecology, 28, 117-133, 1994.

[31] Wojtal A., Witkowski A. and Metzeltin D., The diatom flora of the "Bor na Czerwonem" raised peat-bog in the Nowy Targ Basin (Southern Poland), Fragmenta Floristica et Geobotanica, 44, 167-192, 1999.

[32] Hofmann G., Werum M. and Lange-Bertalot H., Diatomeen im Süßwasser-Benthos von Mitteleuropa. Bestimmungsflora Kieselalgen für die ökologische Praxis, Königstein, Koeltz Scientific Books, 2013.

[33] Ortiz-Lerín R. and Cambra J., Distribution and taxanomic notes of Eunotia Ehrenberg 1837 (Bacillariophceae) in rivers and streams of Northern Spain, Limnetica, 26, 415-434, 2007.

[34] Şahin B., A Study on the Benthic Algae of Uzungöl (Trabzon), Turkish Journal of Botany, 22, 171-189, 1998.

[35] Şahin B., Epipelic and Epilithic Algae of Dağbaşı Lake (Rize-Turkey), Turkish Journal of Botany, 25, 187-194, 2001.

[36] Şahin B. and Akar B., Epipelic and Epilithic Algae of Küçükgöl Lake (Gümüşhane, Turkey), Turkish Journal of Biology, 29, 57-63, 2005.

[37] Alles E., Nörpel-Schemp M. and Lange-Bertalot H., Zur systematik und ökologie charakteristischer Eunotia-Arten (Bacillariophyceae) in elektrolytarmen Bachoberlaufen, Nova Hedwigia, 53, 171-213, 1991.

[38] Şahin B., Algal Flora of Lakes Aygır and Balıklı (Trabzon, Turkey), Turkish Journal of Botany, 24, 35-45, 2000.

[39] Şahin B., Epipelic and Epilithic Algae of the Yedigöller Lakes (Erzurum-Turkey), Turkish Journal of Biology, 26, 221-228, 2002.

[40] Akar B. and Şahin B., Benthic algal flora of Karanlık Lake and diversity of epipelic algae, Fresenius Environmental Bulletin, 15, 48-54, 2006.

[41] Kolaylı S. and Şahin B., Seasonal variations of benthic diatoms with relation to physical and chemical variables in Karagöl Lake (Borçka-Artvin, Turkey), Fresenius Environmental Bulletin, 17, 956-961, 2008. 
Algal Flora and Ecology of the High Mountain Lakes in the Artabel Lakes Nature Park (Gümüşhane, Turkey), I- Bacillariophyta

[42] Şahin B., Akar B. and Bahçeci İ., Species composition and diversity of epipelic algae in Balık Lake (Şavşat-Artvin, Turkey), Turkish Journal of Botany, 34, 441-448, 2010.

[43] Akar B. and Şahin B., Diversity and ecology of benthic diatoms in Karagöl Lake in Karagöl-Sahara National Park (Şavşat, Artvin, Turkey), Turkish Journal of Fisheries and Aquatic Sciences, 17, 15-24, 2017.

Citation: Sophia Bartnova, et.al," Algal Flora and Ecology of the High Mountain Lakes in the Artabel Lakes Nature Park (Gümüşhane, Turkey), I- Bacillariophyta "'International Journal of Advanced Research in Botany (IJARB), vol. 5, no. 2, pp. 25-37, 2019. http://dx.doi.org/10.20431/2455-4316.0502003.

Copyright: (C) 2019 Authors. This is an open-access article distributed under the terms of the Creative Commons Attribution License, which permits unrestricted use, distribution, and reproduction in any medium, provided the original author and source are credited. 